\title{
Le nouveau Moyen Âge psychiatrique
}

\section{The new psychiatric Middle Ages}

\section{T. Haustgen}

(C) Springer-Verlag 2009

Depuis trois ans se multiplient sur les rayons des libraires les ouvrages dénonçant l'état alarmant des soins psychiatriques en France. Ils émanent tantôt de praticiens de terrain $[1,6,10]$, tantôt de journalistes d'investigation [5,7]. Le drame de Pau a délié les langues. Si les causes du mal et les remèdes varient d'un auteur à l'autre, tous s'accordent sur le diagnostic.

Après les professionnels, les «usagers » et leurs familles, le grand public découvre les carences du système de prise en charge des plus vulnérables de nos concitoyens. Faut-il désigner les responsables ? Faut-il gémir sur le bon vieux temps disparu ? Faut-il dénoncer dans une tirade vertueuse la conjonction maléfique du scientisme et de l'économisme? Faut-il invoquer une sempiternelle «crise» de la psychiatrie ? Confrontés aux mêmes difficultés, nos lointains prédécesseurs utilisaient des termes moins édulcorés, n'hésitaient pas à parler d' « anarchie » psychiatrique : «affligeant spectacle » pour Casimir Pinel (le neveu du « grand »), en 1866, que la bataille autour des monomanies et son arrière-plan institutionnel ; « confusion des mots et des idées » pour Arnaud, en 1913, que l'inflation des théories concurrentes et des modèles de référence dans une discipline déjà à la recherche de son introuvable spécificité. Il paraît donc plus intéressant de resituer l'actualité dans la longue durée, à travers un rapide coup de projecteur sur trois périodes clés.

À la fin du XVIII ${ }^{\mathrm{e}}$ siècle, les soins aux malades mentaux se répartissaient, à Paris comme en province, entre HôtelsDieu pour les cas aigus, auxquels était réservé un traitement physique standard, et « hôpitaux généraux » (aucun rapport avec le sens actuel) pour les incurables, peu à peu séparés des mendiants et des délinquants. C'est le second type d'établissement qui vit la naissance de notre spécialité, après la nomination de Philippe Pinel comme médecin-chef des infirmeries de Bicêtre en 1793, puis de La Salpêtrière en 1795. Son traitement moral offrait une alternative au traitement physique de 1'Hôtel-Dieu de Paris. L'« hospice d'aliénés, $[\ldots]$ vaste enclos $[\ldots]$, local spacieux, [...]

T. Haustgen

CMP, 77, rue Victor-Hugo, F-93100 Montreuil, France contrepoids aux égarements de l'esprit par l'attrait et le charme qu'inspire la culture des champs », subdivisant agités, tranquilles et convalescents en quartiers séparés, devait permettre son application à grande échelle. Dès 1802, les malades aiguës étaient admises à La Salpêtrière. Mais ni le Consulat de Bonaparte, ni le ministère libéral de Decazes, destinataire sous la Restauration du mémoire d'Esquirol sur les «Établissements des aliénés » (1818), ni même la monarchie constitutionnelle de Louis-Philippe, après le vote de la loi de 1838, n'entreprirent ces constructions idéales et onéreuses sur l'ensemble du territoire. On se débrouillait avec les locaux disponibles : quartiers d'hospices, dépôts de mendicité, maisons de force, institutions religieuses.

Il faudra attendre le Second Empire et les débuts de la Troisième République pour que la France des usines, du chemin de fer, de l'expansion urbaine et du libre-échange se couvre de ces édifices gigantesques, agricoles, ruraux, vivant en circuit fermé, devenus alors anachroniques. Delasiauve, qui proposait la création d' " asiles communaux » ouverts, de petite taille, proches du domicile des patients (1865), ne sera pas écouté. C'est dans la décennie 1860 qu'apparaissent les premières contestations de la loi de 1838 et les premiers pamphlets antipsychiatriques. Les projets initiaux étaient bien oubliés. Le traitement moral s'était transformé en isolement, travail, règlement intérieur, gestion de masse, parfois en intimidation et coercition.

À la fin du XIX ${ }^{\mathrm{e}}$ siècle, l'échec de l'asile était patent. La Belle Époque aurait dû l'être aussi pour la psychiatrie. Elle voit en effet l'émergence de nombreuses propositions

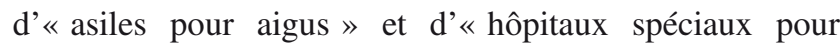
délirants », fonctionnant en dehors du cadre de la loi de 1838. Marandon de Montyel conduit dès 1888, à VilleEvrard, une expérience sans lendemain d' « hospitalisation des aliénés par la méthode de liberté ». Régis à Bordeaux, Gilbert Ballet à 1'Hôtel-Dieu de Paris créent, autour de 1900, des services ouverts de « délirants ». Neurasthéniques et hystériques, y compris celles de Charcot, séjournent à partir de 1870 sans y être internés dans les services de maladies nerveuses de l'Assistance publique. Edouard Toulouse publie ses premiers projets en 1896, en s'appuyant sur une conjonction baroque de scientisme, 
d'eugénisme, de préoccupation pour les « petits mentaux » et de rejet des incurables, considérés comme «non-valeurs sociales définitives $»$.

Cette fois encore, c'est seulement une génération plus tard que ces «services libres» commencent à se concrétiser, dans un pays exsangue et ruiné par le premier conflit mondial. Le monde s'est transformé, non plus sous l'effet d'une révolution industrielle comme en 1850, mais sous celui d'une crise économique majeure. Dans une perspective plus eschatologique que réaliste, Toulouse veut séparer radicalement ses services ouverts des asiles, voués à péricliter, au moins sur le plan de la sémantique. Mais ses collègues aliénistes se méfient et conservent leurs lits. La mutation terminologique de l'asile en hôpital psychiatrique et la circulaire Rucart sur les soins extrahospitaliers ne précèdent que de trois ans l'hécatombe de 1940-1944 [3].

Après 1945, le mouvement désaliéniste, conduit non plus à l'échelle des seuls services ouverts mais sur l'ensemble du parc hospitalier, aboutit à la sectorisation dont, symboliquement en plein milieu des Trente Glorieuses, la circulaire de 1960 pose les fondements. Expansion économique, croissance ininterrompue, plein emploi, sécurité sociale et mise sur le marché des premiers psychotropes autorisent tous les espoirs. Mais, comme en 1838, comme à la Belle Époque, la mise en pratique se fait attendre, les pouvoirs publics renâclent. La situation se débloque au début de la décennie 1970. La psychiatrie connaît alors des années fastes, marquées par une mutation institutionnelle et universitaire. Nous en conservons quelques beaux restes, surtout dans le domaine de la démographie professionnelle - médicale, mais hélas plus infirmière. Les orages futurs ne tardent pas à s'annoncer.

L'arrêté Boulin de 1972 et la circulaire Poniatowski de 1974 encadrent le premier choc pétrolier. Lorsque la sectorisation fait l'objet d'un texte de loi en 1985, le chômage a explosé, les «nouveaux pauvres » ont fait leur apparition, tandis que le rapport Demay (avant d'autres) recycle les utopies d'Edouard Toulouse sur le « dépérissement $»$ des structures hospitalières.

Un ministre de la Santé annonce, dès 1980, la suppression de 40000 lits de psychiatrie. Le programme s'est réalisé au-delà de ses espérances. C'est la moitié des 100000 lits de la spécialité qui a été fermée en une génération, d'abord sous l'euphémisme rassurant du « redéploiement ». À l'ère de l'exclusion et de la précarité, on a troqué un risque de chronicisation institutionnelle contre une quasi-certitude de clochardisation hors les murs pour de nombreux malades. Tandis qu'on doit déchanter quant à la supposée supériorité des «nouveaux » antipsychotiques - clozapine exceptée - par rapport aux anciennes molécules [2].

Il faut s'y résigner : on ne guérit pas tout en psychiatrie, un contingent incompressible de patients reste au-delà des ressources de l'ensemble des thérapeutiques. Épilogue provisoire : un autre ministre de la Santé annonce, 25 ans après celui de 1980, un moratoire sur les fermetures de lits...

Des quatre espèces de la nosographie pinélienne, seules relèvent encore de l'hospitalisation manie et mélancolie (au sens antique, incluant les délires). Familles, maisons de retraite, IME et secteur médicosocial se partagent (avec quelle pénurie soignante !) démence et idiotisme - c'est-àdire schizophrénies déficitaires, pathologies d'involution sénile et présénile, autisme et oligophrénies. En revanche, victimes de catastrophes naturelles, harcelés au travail, délinquants sexuels, adeptes des «nouvelles » addictions et autres prétendues «nouvelles» pathologies intéressent beaucoup la psychiatrie. Chaque époque a ses petits mentaux. Dans ces conditions, il ne faut pas s'étonner que gériatres et neurologues monopolisent les recherches sur la maladie d'Alzheimer ou que professionnels du handicap et de l'éducatif se saisissent du traitement de l'autisme. Itard contre Pinel, Edouard Seguin contre Félix Voisin, programme TEACCH contre psychothérapie : le décor change, mais le scénario reste le même.

Le secteur s'est complété du réseau. La sociothérapie de Le Guillant a accompli sa mue en réhabilitation, remédiation et psychoéducation. L'hygiène mentale d'Edouard Toulouse est devenue santé mentale. Chaslin jugeait déjà, en 1914, le terme médecine mentale bien plus harmonieux aux délicates oreilles françaises que le rude vocable germanique de psychiatrie, décidément indigne d'une « langue bien faite » [4]. Pourtant, mens latine contre psyche grecque, rien n'y fait ! Les soins à deux vitesses entre services hospitaliers, devenus pratiquement des services d'urgence, et structures de long séjour restituent la dichotomie prépinelienne entre Hôtels-Dieu et " hôpitaux généraux ». Rançon des suppressions de lits et des pénuries infirmières, le pavillon unique par secteur marque le retour, dans la pratique sinon dans les textes, des services fermés, avec leur encombrement, leurs serrures et leurs chambres d'isolement. Ainsi, la psychiatrie du XxI ${ }^{\mathrm{e}}$ siècle, en dépit de ses énormes progrès techniques, a-t-elle rétabli la cohabitation dans le même espace des agités et des tranquilles, après la ségrégation entre curables et incurables. Double aberration de l'appareil d'assistance aux malades mentaux sous l'Ancien Régime, combattu au XIX ${ }^{\mathrm{e}}$ siècle par les promoteurs de l'asile, $\mathrm{au} \mathrm{xx}^{\mathrm{e}}$ par les pionniers du secteur.

En marge des structures de soins s'offre le spectacle du fou médiéval errant dans la ville à la recherche d'un asile (au sens étymologique), dont à l'ère des multimédias le petit écran nous présente l'exhibition filmée. À quoi bon l'hospitaliser ? «Ils » ne le garderaient pas, commentent les âmes charitables [11].

L'augmentation de $75 \%$, en dix ans, des tentatives de suicide dans le métro parisien apporte une touche underground à ce tableau digne de Jérôme Bosch [8]. 
Fin de la dictature de la raison cartésienne qui réjouira les vétérans de l'antipsychiatrie et les mânes de Michel Foucault, dont la célébration actuelle par l'intelligentsia, sans aucune distance critique, confine à 1'hagiographie. Les augures qui invoquent sa «modernité » à tout propos perçoivent-ils l'ironie de leur constat ?

C'est donc bien, toute démagogie décliniste mise à part [9] un Moyen Âge au sens propre que la psychiatrie traverse actuellement : une époque intermédiaire, comme le Second Empire et l'entre-deux-guerres, durant laquelle persistent quelques vestiges de l'époque précédente, au milieu de « nouveautés» ou prétendues telles qui les submergent. Cette spécialité médicale est-elle condamnée à un perpétuel train de retard, à la succession de cycles réformateurs avortés, suivis de désillusions ? On commence à apercevoir le ressort profond de ces grands désarrois à répétition de la psychiatrie : un décalage entre ses projets et les mutations de la société au moment où ils entrent en application, une incapacité à s'adapter aux changements de conjoncture - preuve s'il en était besoin $\mathrm{du}$ lien indissoluble entre le social et le psychiatrique. Le marasme économique présent nous rapproche sans doute plus des années 1930 que de Napoléon III. On peut toujours se consoler en se comparant à l'Italie ou aux États-Unis. Mais ce ne sont pas des aumônes distribuées ici ou là par les pouvoirs publics qui amèneront une renaissance psychiatrique.

\section{Références}

1. Bokobza H (dir) (2006) La psychiatrie en péril. Erès, Ramonville-Saint-Agne

2. Bottéro A (2008) Un autre regard sur la schizophrénie. Odile Jacob, Paris

3. Bueltzingsloewen (von) I (2007) L'hécatombe des fous. La famine dans les hôpitaux psychiatriques français sous l'Occupation. Aubier, Paris

4. Chaslin P (1914) La «psychiatrie » est-elle une langue bien faite? Rev Neurol XXVII(1):16-23

5. Coupechoux P (2006) Un monde de fous. Comment notre société maltraite ses malades mentaux. Seuil, Paris

6. Descombey JP (2007) La psychiatrie sinistrée. L'Harmattan, Paris

7. Dufau S (2006) Le naufrage de la psychiatrie. Albin Michel, Paris

8. Le Point, $1^{\text {er }}$ juin 2006.

9. Minc A (1993) Le nouveau Moyen Âge. Gallimard, Paris

10. Quesemand-Zucca S (2007) Je vous salis ma rue. Stock, Paris

11. Urgences psychiatriques, des lieux à haut risque. M 6, Enquête exclusive, 27 avril 2008 